\title{
Needle-Free Administration of Botulinum Toxin for Primary Focal Hyperhidrosis: Initial Experience
}

\author{
Artzi 0* \\ Department of Dermatology, Tel Aviv Medical Center, Israel
}

*Corresponding author: Ofir Artzi, MD, Department of Dermatology, Tel Aviv Medical Center, 6 Weizman Street, Tel Aviv, 6423906, Israel, Tel: +972-3- 036973356; Fax: +972-3- 6974810; E-mail: ofira@tlvmc.gov.il

\section{Research Article}

Volume 2 Issue 3

Received Date: August 13, 2017

Published Date: September 02, 2017

DOI: $10.23880 /$ cdoaj-16000131

\section{Abstract}

Local intradermal injections of botulinum toxin (BTX) effectively provide symptom relief of primary focal hyperhidrosis (PFHH), but they can be painful and a deterrent for repeated treatments. The Enerjet system delivers therapeutic substances into the dermis via a high-pressure liquid jet. In this proof-of-concept trial, BTX was administered by the EnerJet system without anesthesia. The pre- and 4 weeks' post-treatment disease severity and symptom burden were assessed [Hyperhidrosis Disease Severity Scale (HDSS), the Dermatology Life Quality Index (DLQI) and Minor starchiodine tests]. All 11 patients had severe PFHH. The number of required shots ranged between 50-90 for the bilateral axillary areas and 75-100 for the palmar areas. Total reduction of DLQI and HDSS scores at 4 weeks post-EnerJet system treatment indicated improvement in symptom burden and quality of life. BTX delivery by the EnerJet system may be a simple and effective alternative to conventional BTX injections.

Keywords: Palmar Hyperhidrosis; Botulinum toxin; Needle-free injection

Abbreviations: BTX: Botulinum Toxin; PFHH: Primary Focal Hyperhidrosis; DLQI: Dermatology Life Quality Index; HDSS: Hyperhidrosis Disease Severity Scale

\section{Introduction}

Primary focal hyperhidrosis (PFHH) refers to the idiopathic medical condition of excessive sweating in certain areas, most commonly the axillary, palmar, and plantar regions. The condition affects the patient's quality of life and causes considerable physical discomfort and psychosocial impairment [1]. In addition to the multiple topical agents recommended as the first line of treatment, intradermal injections of botulinum toxin (BTX) appear to be an effective method of symptom relief. However, the administration of BTX through local injections can be extremely painful, and that might discourage the patients from undergoing repeated treatments. While injecting the palmar or plantar areas, pain-control methods (topical analgesics, regional anesthesia, peripheral nerve blockade and cryo-analgesia) are required, raising the cost of the procedure [2,3].

The needle-free injection technology of the Enerjet system (PerfAction Technologies, Rehovot, Israel) is designed to administer and laterally disperse different therapeutic substances into the dermis via a highpressure liquid jet $[4,5]$. In this proof-of-concept trial we administrated BTX via the EnerJet system.

\section{Materials and Methods}

The study included 11 patients $(7$ males and 4 females, age range 32-47 years) who had not been adequately managed by topical agents for axillary $(n=7)$ and palmar 
$(n=4)$ primary hyperhidrosis and were treated with the EnerJet system. Five out of those 11 patients had received BTX needle injections for the treatment of PFHH in the past. All patients signed an informed consent. In this trial, we used on a botulinum toxin $A$ (Botox $囚$ ) at the manufacturer's recommended dose for the treatment of PFHH of the axillary and palmar regions. A total of $100 \mathrm{U}$ of Botox $\AA$ diluted with $7 \mathrm{ml}$ of bacteriostatic saline were administrated into both palms (50U per palm). The administration spots were evenly distributed over the treated area in a grid of multiple sites approximately 1-2 $\mathrm{cm}$ apart, identical to the needle injection approach. The average operational pressures varied from 40\% (3.04 bar) in the axillary area to $50 \%$ (3.57 bar) in the palmar area, which can be explained by the differences in skin permeability in these regions. The resulting skin papule represented sufficient intradermal penetration of the drug (Figure 1). Two additional sites in every treated area were injected with a sterile 30-gauge needle for comparison of the pain level. The participants were asked to evaluate the pain caused by EnerJet and by needle administration on a 0-10 Numeric Pain Rating Scale.

Prior to treatment and 4 weeks' post-treatment, the disease burden and severity of disease were assessed by the Dermatology Life Quality Index (DLQI) for hyperhidrosis and the Hyperhidrosis Disease Severity Scale (HDSS). The DLQI consists of 10 items on leisure, personal relationships, daily activities, and treatment. The highest score indicates the highest level of impairment in the patient's quality of life. The HDSS quantifies oversweating as mild (1), moderate (2) and severe (3-4). In addition, pre-and post-treatment Minor starch iodine tests were performed to evaluate the change of hyperhidrosis.

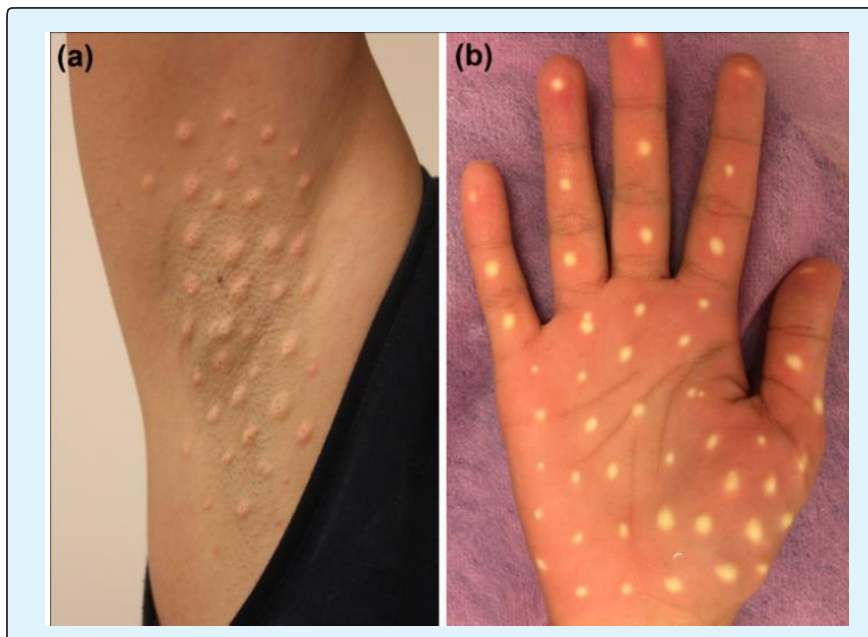

Figure 1: Skin papule in axillary and palmar areas following EnerJet administration.

\section{Results}

Demographic and clinical baseline data of the participants are summarized in Table 1. The baseline HDSS demonstrated that all patients had severe PFHH, with an average of $3.27 \pm 0.64 / 3.18 \pm 0.6(\mathrm{~L} / \mathrm{R})$ for the sweating regions. Each participant underwent a single treatment session. The number of shots required to cover the treated area ranged between 50-90 for the bilateral axillary areas and 75-100 for the palmar areas.

\begin{tabular}{|c|c|c|c|c|}
\hline Case & Gender & Treated area & DLQI & HDSS (left/right) \\
\hline 1 & Male & Underarms & 29 & $2 / 3$ \\
\hline 2 & Male & Underarms & 15 & $4 / 4$ \\
\hline 3 & Male & Underarms & 14 & $3 / 3$ \\
\hline 4 & Male & Underarms & 10 & $4 / 4$ \\
\hline 5 & Male & Underarms & 9 & $4 / 3$ \\
\hline 6 & Female & Underarms & 22 & $3 / 3$ \\
\hline 7 & Female & Underarms & 7 & $3 / 3$ \\
\hline 8 & Female & Palms & 13 & $4 / 4$ \\
\hline 9 & Female & Palms & 18 & $3 / 2$ \\
\hline 10 & Male & Palms & 18 & $3 / 3$ \\
\hline 11 & Male & Palms & 16 & $3 / 3$ \\
\hline
\end{tabular}

Table 1: Demographics and Baseline Data.

DLQI, Dermatology Life Quality Index; HDSS, Hyperhidrosis Disease Severity Scale. 
Pre-treatment local anesthesia was not provided, and all the patients tolerated the treatment well. The average pain score was 3.1 (2-7) for the axillary region and 3.5 (2-
5) for the palmar region. In contrast, the average pain scores for the needle-injected sites were 5.3 and 8.5, respectively (Table 2 ).

\begin{tabular}{|c|c|c|c|c|c|}
\hline Case & Treated area & Procedure-related pain & DLQI & $\begin{array}{c}\text { HDSS } \\
\text { (left/right) }\end{array}$ & Change in appearance (left/right) $^{*}$ \\
\hline 1 & Underarms & 2 & 8 & $2 / 2$ & $3 / 4$ \\
\hline 2 & Underarms & 7 & 8 & $2 / 3$ & $3 / 1$ \\
\hline 3 & Underarms & 5 & 6 & $2 / 2$ & $1 / 2$ \\
\hline 4 & Underarms & 2 & 1 & $4 / 2$ & $4 / 4$ \\
\hline 5 & Underarms & 2 & 9 & $2 / 1$ & $1 / 1$ \\
\hline 6 & Underarms & 2 & 12 & $3 / 2$ & $4 / 1$ \\
\hline 7 & Underarms & 2 & 3 & $2 / 1$ & $2 / 3$ \\
\hline 8 & Palms & 4 & 5 & $2 / 2$ & $3 / 2$ \\
\hline 9 & Palms & 2 & 10 & $1 / 2$ & $2 / 1$ \\
\hline 10 & Palms & 3 & 10 & $2 / 2$ & $3 / 4$ \\
\hline 11 & Palms & 5 & 12 & $2 / 2$ & $2 / 2$ \\
\hline
\end{tabular}

Table 2: Treatment Outcomes.

DLQI, Dermatology Life Quality Index; HDSS, Hyperhidrosis Disease Severity Scale.

${ }^{*}$ Graded by 2 dermatologists. Change in appearance was graded as a quartile grading scale: $0=$ no change, $1=a$ minor change of $<25 \%, 2=$ a moderate change of $25-50 \%, 3=$ a major change of $50-75 \%$, and $4=$ the absence or near-absence of sweating.

The DLQI scores at 4 weeks following treatment by the EnerJet system demonstrated a reduction in total score from the baseline average of $15 \pm 7$ to the post-treatment score of $7.63 \pm 3.55$ for the sweating areas. The posttreatment HDSS scores also decreased to an average of $2.18 \pm 0.75 / 1.9 \pm 0.53(\mathrm{~L} / \mathrm{R})$.

Images of the follow-up Minor starch-iodine tests were blindly assessed and compared to the baseline independently by two certified dermatologists. Change in appearance was graded as a quartile grading scale: $0=$ no change, $1=$ a minor change $(<25 \%), 2=$ a moderate change (25-50\%), $3=$ a major change (50-75\%) and $4=$ the absence or near-absence of sweating. The left and right sides were graded separately, and both demonstrated a decrease in the sweat over activity in all patients by an average of $2.17 \pm 1.21 / 2.27 \pm 1.27(\mathrm{~L} / \mathrm{R})$ for the sweating regions (Figures $2 \& 3$ ). Agreement between the two reviewers was not assessed.

All patients expressed satisfaction with the treatment procedure and the results.
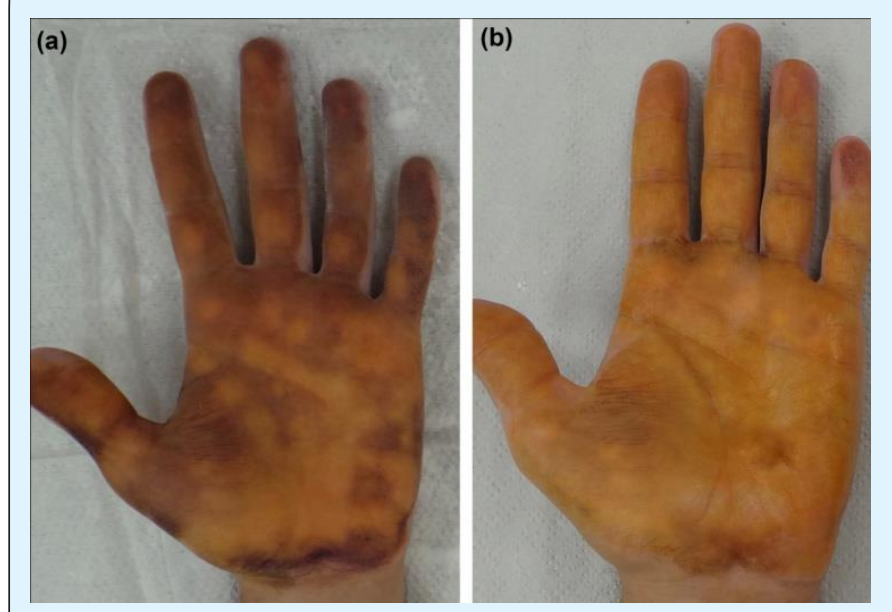

Figure 2: Palmar hyperhidrosis (45-yo male). Minor test before treatment (A) and at follow-up (B). 


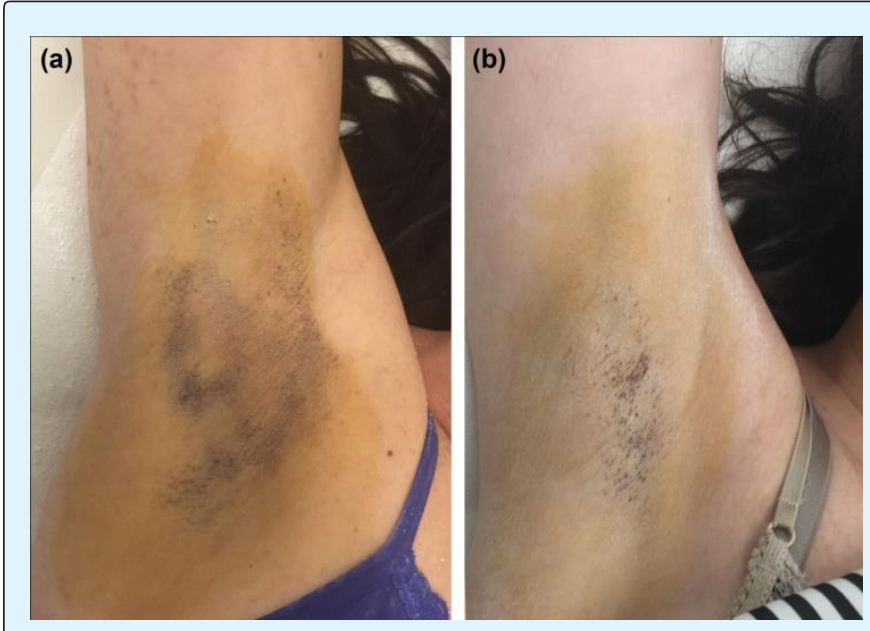

Figure 3: Axillary hyperhidrosis (36-yo female). Minor test before treatment (A) and at follow-up (B).

\section{Discussion}

$\mathrm{PFHH}$ is considered a debilitating disorder that influences the affected individual's physical, psychological, and social well-being. Epidemiology surveys reported the prevalence of axillary hyperhidrosis in $1.4 \%$ and palmar hyperhidrosis in $4.59 \%$ of the population [6,7]. BTX injections have shown success for the treatment of PFHH. BTX inhibits pre-synaptic release of acetylcholine which results in chemical denervation of the glands and temporary cessation of hyperhidrosis. The desired injection depth for BTX administration is the deep dermis, at or near the junction with the subcutaneous tissue [8].

Administering BTX by the needle-free EnerJet system provides technology-controlled uniformity and consistency in penetration depth and distribution of the material. Once having reached the targeted skin layer, the medication is distributed spherically in approximately 1 $\mathrm{cm}^{2}$ areas.

The objective and subjective measures in our study confirmed a decrease of the symptoms (HDSS) and improvement in the quality of life (DLQI) for all participants, as well as a reduction in glandular sweat activity in the treated area (Minor starch-iodine test). The treated areas did not require any pre-treatment anesthesia. All patients expressed satisfaction with the procedure and with the results, reporting that the injection pain was much lower compared to the control needle injections. When compared to the efficacy of previous BTX needle injections, eight participants rated the current results of EnerJet treatment as being "the same" or "somehow better", based on a scale from 1 (worse) to 5 (much better). Ten out of the 11 study patients confirmed that they would repeat the EnerJet treatments again.

Unlike the needle injections, which are time-consuming and rely on high levels of operator skill to achieve targeted deposition of BTX in the dermis, the administration of BTX through the needle-free EnerJet system can be quicker and less bothersome for both the physician and the patient [9]. No adverse events associated with either the EnerJet administration procedure or with BTX injections had being reported.

This study has some limitations, and the results must be interpreted with some caution. It was conducted on a small number of patients who were followed during a period of several weeks. Further studies are required on a larger number of patients and with a longer follow-up period in order to assess the statistical significance of the results and to explore their duration. The efficacy of administering BTX by the needle-free EnerJet system, in terms of severity and disease burden should be compared to the efficacy of the conventional BTX needle injections. In addition, presence of the fluid residuals on the skin after each administration may raise some questions about the extent of actual skin penetration, thus calling for additional investigation to measure the amounts of those residuals.

Nevertheless, it is our considered opinion that the administration of BTX by means of the EnerJet system can be a simple, safe and effective method of computerconsistent drug delivery. It might be an alternative to the conventional administration by injections, especially for hyperhidrosis patients with low pain tolerance for palmar or plantar needle injections. A controlled comparative clinical trial on a larger population of patients is warranted in order to optimize the treatment regimen and dosing and validate these preliminary findings.

\section{References}

1. Grunfeld A, Murray CA, Solish N (2009) Botulinum toxin for hyperhidrosis: a review. Am J Clin Dermatol 10(2): 87-102.

2. Kang A, Burns E, Glaser DA (2015) Botulinum toxin A for palmar hyperhidrosis: associated pain, duration, and reasons for discontinuation of therapy. Dermatol Surg 41(2): 297-298. 
3. Alodeani EA (2016) Botulinum toxin type A: an effective, safe and minimally invasive treatment option of axillary and palmar hyperhidrosis. Int J Pharm Pharm Sci 8(7): 237-240.

4. Lee JW, Kim BJ, Kim MN, Lee CK (2010) Treatment of acne scars using subdermal minimal surgery technology. Dermatol Surg 36(8): 1281-1287.

5. Mashiko T, Abo Y, Kuno S, Yoshimura K (2015) A novel facial rejuvenation using pneumatic injection of non-cross-linked hyaluronic acid and hypertonic glucose solution. Dermatol Surg 41(6): 755-758.

6. Strutton DR, Kowalski JW, Glaser DA, Stang PE (2004) US prevalence of hyperhidrosis and impact on individuals with axillary hyperhidrosis: results from a national survey. J Am Acad Dermatol 51(2): 241-248.
7. Tu YR, Li X, Lin M, Lai FC, Li YP, et al. (2007) Epidemiological survey of primary palmar hyperhidrosis in adolescent in Fuzhou of People's Republic of China. Eur J Cardiothorac Surg 31(4): 737-739.

8. Glaser DA, Hebert AA, Pariser DM, Solish N (2007) Palmar and plantar hyperhidrosis: best practice recommendations and special considerations. Cutis 79(5): 18-28.

9. Torrisi BM, Zarnitsyn V, Prausnitz MA, Anstey A, Gateley C, et al. (2013) Pocketed microneedles for rapid delivery of a liquid-state botulinum toxin $\mathrm{A}$ formulation into human skin. J Control Release 165(2): 146-152. 